awareness is necessary to ensure that ACPs are reviewed appropriately.

\section{WHO WOULD KNOW? EMBEDDING FUTURE CARE PLANNING FOR ALL PATIENTS APPROACHING THE END OF THEIR LIVES}

Clare E Finnegan. Lancashire and South Cumbria NHS Foundation Trust

10.1136/spcare-2020-PCC.78

Background Future Care Planning (FCP) empowers individuals to receive the care and treatment they choose towards the End of Life (EoL). Developing personal Advance Care Plans (ACPs) and Anticipatory Clinical Management Plans (ACMPs) improves patient and family satisfaction and reduces burdensome' treatments yet is not universally offered. An evaluation of current FCP across a locality was undertaken to establish current practice, explore barriers to implementation and guide future work.

Methods

1. Case note review of patients known to specialist palliative care services(SPCS) for evidence of FCP and informationsharing between organisations $(n=52)$

2. Review of ACMP content and information-sharing between organisations $(n=50)$

3. Health-care professional survey of knowledge, experience and expectations of FCP $(n=39)$

Results Preferred Place of Care was documented for $85 \%$ of patients known to SPCS, but there was limited documented evidence of other forms of FCP (uDNACPR:38\%, ACMP:2\%, ADRT/LPA:0). FCP undertaken by SPCS was rarely available in hospital records. The number of ACMPs is increasing (49 $(2017 / 18)$ c.f. 198 (2018/19)), predominantly for care home residents, with $60 \%$ developed by newly appointed frailty practitioners. They consistently included key EoL topics but only 38\% were available within hospital records. Staff had limited knowledge and experience of FCP, and the processes for recording and sharing patients wishes. They felt more training and more time would enable them to facilitate more ACP conversations

Conclusions Not all patients are currently offered an ACP conversation. Although information packs are available further promotion is required to ensure they are given. There is a need to continue current network-wide ACP training; additional targeted support for those in key roles has been introduced. Collaborative working between SPCS, frailty, acute and primary care teams is ongoing to ensure a consistent approach, and shared documentation and processes to ensure patients wishes are respected as they move between care settings.

\section{ANTICIPATORY PRESCRIBING AND USE OF SYRINGE DRIVERS - CROSS BOUNDARY AUDIT OF PRACTICE}

Andrew Fletcher, Kathryn Woods, Lily Chan, Grace Nichols, Lynn Kelly, Linda Dewhurst, Maria Debattista. Lancashire Teaching Hospitals NHS Foundation Trust, St Catherine's Hospice Preston, Lancashire Care Foundation Trust

10.1136/spcare-2020-PCC.79

Background Anticipatory prescribing and use of syringe drivers at end of life is now established practice. A national report outlined the need to ensure safe, appropriate and individualised prescribing for patients at end of life, considering available guidance to aid decision making. Within our locality we deemed it vital to demonstrate we are complying with guidance.

Aims To ensure safe and effective care of patients at end of life, with an individualised approach to decisions and compliance with guidelines, policies and procedures.

Methods Questions were developed through collaborative discussion and data collection tool produced. Retrospective audit cross settings was undertaken based on place of death to avoid duplication.

Results 71 cases included for full analysis. Largest proportion in Hospice. Spread of gender, age, diagnosis. Substantial data was collected on core drugs at end of life. Using agitation as an example, 93\% had an individualised approach to prescribing: all had a dose and route; 1 no frequency although this was felt to be a documentation error; $31 \%$ had a maximum dose; 93\% had an appropriate dose adjustment for renal impairment. In the last 24 hours of life the total dose of midazolam: Hospice median $1.25 \mathrm{mg}$ and mean $8.2 \mathrm{mg}$; hospital $0 \mathrm{mg}$ and $1.7 \mathrm{mg}$ and community $0 \mathrm{mg}$ and $5 \mathrm{mg}$ respectively. Similar results were found for other drugs in terms of quality of prescriptions and individualised nature of decision making.

Conclusion Individualised approach to prescribing was evident overall, with a high standard of prescribing. Some incomplete prescription although felt due to data collection errors. Doses of medications in line with network guidelines. Need to improve discussion with patients and families regarding medications, syringe drivers and side effects. The three organisations involved developed individual action plans including improvements in documentation and where electronic prescribing had commenced, embedding this.

\section{IMPROVING IDENTIFICATION AND RECORDING OF THE GOLD STANDARDS FRAMEWORK (GSF) WITHIN RUSSELLS HALL HOSPITAL, DUDLEY}

Hannah Gay, Kate Hall, Joanne Bowen. Dudley Group NHS Foundation Trust

\subsection{6/spcare-2020-PCC.80}

Introduction Measuring performance in End of Life Care within an acute setting is a complex and difficult task. GSF implementation within a hospital setting can improve quality of care, patient satisfaction and an increase in advance care planning. This study aims to show how by developing an internal hospital application and protocol for GSF patients, improved data collection can be transformed from data into intelligence and impact on delivery of high quality, individualised end of life care.

Methods Most wards within Dudley had different ways of capturing GSF identified patients and recorded information manually. Informatics and the Specialist Palliative Care team developed an internal application to monitor all patient admissions against a GSF outcome and to provide additional information such as preferred place of care and ACP. This provided the ward with a visual management tool, easily identifying end of life patients and the ability to capture essential end of life metrics.

Results Since the launch of this application, analysis of the data has demonstrated an increase in the percentage of adult 
inpatients assessed for GSF and currently using this information for targeted education and learning. As of the 30th September 2019, the three front runner wards had a combined assessment rate of $90.2 \%$ Through this log, other metrics broken down by ward include identification rate, percentage of patients who achieve their preferred place of care and how many have an individualised plan of care in place or offered ACP.

Conclusion Initial results indicate that having an electronic centralised database for monitoring GSF outcomes provides a platform to scrutinise key metrics for end of life care, which enable the wards and specialist palliative care team to ensure high quality end of life care is achieved.

\section{CRITICAL CARE AND PALLIATIVE CARE: A LIASION QIP}

N Gunawardena, J Bichard. North Middlesex Hospital

\subsection{6/spcare-2020-PCC.81}

Background and Introduction The NMUH Specialist Palliative Care Team (SPCT) were finding that patients discharged from Critical Care with a treatment escalation plan 'Not for readmission to ITU/HDU' were experiencing high levels of suffering on the wards for several days prior to SPCT review. They were also dying within the same hospital admission and not reaching their preferred place of care. The team proposed the idea of having all patients from this group referred at point of CRC discharge which would give these patients faster access to expert symptom control, advanced care planning and transfers to preferred place of care.

Method A baseline audit conducted on all patients discharged from CRC from 1/1/18-30/11/18 revealed that only $14 \%$ of patients with a TEP form 'Not for admission to ITU/HDU' were referred to SPCT at point of CRC discharge. Over 7 months several interventions were implemented through monthly PDSA cycles to improve the referral rate. This included emails sent to CRC staff, palliative care teaching at the CRC educational sessions and training of the critical care outreach team in making SPCT referrals.

Results Overall there was an improvement of referral rate of this group of patients to SPCT from $14 \%$ to $70 \%$ during the 7 months where the interventions took place. Moreover, the increasing collaboration between the two teams through this QIP culminated in the introduction of a palliative care representative at the weekly CRC MDT meeting.

Conclusions While an overall improvement in referral rate to SPCT was established it remains to be seen whether patient outcomes have improved due to this change. Thus a case note review will be conducted to assess this. Rotation of juniors in CRCmeans that the educational interventions need to be recycled biannually to keep up referral rate.

\section{IMPROVING DISCUSSIONS AROUND TREATMENT ESCALATION DECISIONS ON A NEUROSURGICAL WARD}

E Haire, G Kimpton, K Whitehouse, A Williams, S Srivastava. North Bristol Trust

\subsection{6/spcare-2020-PCC.82}

Background It is becoming increasingly accepted that discussion and documentation of patient wishes and clinical decisions regarding treatment escalation planning are an essential part of patient care. Following the Tracey judgement, it became a legal requirement, rather than a recommendation, for doctors to involve patients and their families in DNAR decisions. A previous audit in the neurosurgical department found a high prevalence of patients at risk of deterioration with little evidence of attempt to discuss or document treatment escalation decisions (TEDs).

Aims

- To improve the documentation of TEDs in the neurosurgical department

- To improve confidence and communication about TEDs within the multi-disciplinary team

Methods We conducted surveys of the multi-disciplinary team within the neurosurgical department to gain a better understanding of their opinions about the current discussion and documentation of TEDs in the department. These contained a mixture of rating scales and free text answers. Following this we engaged senior staff in plans to improve this by introducing these discussions to governance meetings and educated all members of the ward using e learning and tea trolley teaching.

Results Amongst 18 healthcare professionals, 12 felt patients did not have clear TEDs. Ten felt discussions were not appropriately timed. Response themes included feeling unsupported, poor timing of conversations and low quality documentation. Following this a consensus decision from the consultant body mandated documentation of TEDs in patients presenting with chronic subdural haematomas. We have worked closely with the national ReSPECT programme and established the neurosurgical ward as a lead for the introduction of the new form.

Conclusion The neurosurgery department is now at the forefront of the move to improving discussions and documentation about patient wishes in the Trust. Additionally, we have opened the conversation within the multi-disciplinary team and provided training and education about the importance of TEDs.

\section{ABSTRACT WITHDRAWN}

\section{SYSTEMATIC REVIEW OF PROGNOSTIC VARIABLES ASSOCIATED WITH MORTALITY IN ADULT PATIENTS WITH HEART FAILURE}

Sophie Hancock, Anna Oriani, Miriam J Johnson, Una Macleod, Amy Gadoud. Lancaster University, Oncology Institute of Southern Switzerland, Hull York Medical School

\subsection{6/spcare-2020-PCC.83}

Background Heart failure is a serious life limiting condition and patients can have a high symptom burden, reduced quality of life and increased unscheduled hospital admissions. Despite guidance advocating a palliative care approach in advanced heart failure, the variable disease trajectory leads to difficulty in predicting prognosis, which acts as a barrier to considering palliative care for these patients.

Aims This systematic review explores which clinically relevant variables are associated with a poor prognosis in heart failure and examines if it is possible to determine which of these variables best predict mortality. 